\title{
Simulations of Coulomb systems confined by polarizable surfaces using periodic Green functions
}

\author{
Alexandre P. dos Santos, ${ }^{1,2, a)}$ Matheus Girotto, ${ }^{1, b)}$ and Yan Levin ${ }^{1, c)}$ \\ ${ }^{1}$ Instituto de Física, Universidade Federal do Rio Grande do Sul, Caixa Postal 15051, CEP 91501-970 \\ Porto Alegre, RS, Brazil \\ ${ }^{2}$ Fachbereich Physik, Freie Universität Berlin, 14195 Berlin, Germany
}

(Received 24 July 2017; accepted 25 October 2017; published online 10 November 2017)

\begin{abstract}
We present an efficient approach for simulating Coulomb systems confined by planar polarizable surfaces. The method is based on the solution of the Poisson equation using periodic Green functions. It is shown that the electrostatic energy arising from the surface polarization can be decoupled from the energy due to the direct Coulomb interaction between the ions. This allows us to combine an efficient Ewald summation method, or any other fast method for summing over the replicas, with the polarization contribution calculated using Green function techniques. We apply the method to calculate density profiles of ions confined between the charged dielectric and metal surfaces. Published by AIP Publishing. https://doi.org/10.1063/1.4997420
\end{abstract}

\section{INTRODUCTION}

Efficient simulations of charged systems are of fundamental importance for physics, chemistry, and biology. Because of the long range nature of the Coulomb force, one cannot use simple periodic boundary conditions which are sufficient for systems with short range interactions. Instead one is forced to construct an infinite set of replicas of the original system so that a particle in the main simulation cell interacts with all the other particles in the cell, as well as with all the periodic replicas. To efficiently sum over the replicas of the system, Ewald summation methods have been developed. ${ }^{1-8}$ Originally, the Ewald summation was used to calculate the bulk energy of ionic crystals and, in particular, the Madelung constant. The Ewald summation is based on the separation of the Coulomb potential into long- and short-range contributions. The short-range part can be treated using the usual periodic boundary conditions, while the long-range part can be efficiently summed in the Fourier space. Unfortunately, the method loses much of its usefulness when the full 3D symmetry is broken, which is the case when interfaces are present. This is due to the appearance of special functions in the two-dimensional Fourier transform, leading to slow convergence of the lattice sums. ${ }^{9-11}$ Notwithstanding this, there are many important systems with a broken symmetry: ionic liquids at electrified interfaces, ${ }^{12-17}$ charged nanopores, ${ }^{18-20}$ and nanoconfined electrolytes, ${ }^{21-23}$ just to cite a handful of examples. These systems can present new phenomena, such as like-charged attraction ${ }^{24-27}$ and charge reversal, ${ }^{28-30}$ which are hard to describe analytically,${ }^{31}$ hence the importance of fast simulation methods. To overcome the difficulty of using the 2D Ewald summation, a number of

\footnotetext{
a)Electronic mail: alexandre.pereira@ufrgs.br

b)Electronic mail: matheus.girotto@ufrgs.br

c)Electronic mail: levin@if.ufrgs.br
}

approaches have attempted to extend the efficient 3D Ewald summation method to systems with slab geometry. ${ }^{22,32-34}$ These approaches rely on the introduction of a sufficiently large vacuum region between the undesired replicas to diminish their interaction in the non-periodic direction. To account for the conditional convergence of the lattice sums, the Ewald summation must be performed in a "plane-wise" manner, leading to an additional correction to the usual 3D Ewald energy. The method was shown to be very efficient for simulating systems with reduced symmetry. The difficulty, however, arises when the simulation cell is bounded by the polarizable surfaces such as metal electrodes or phospholipid membranes. If there is only one polarizable surface present, it is straightforward to extend the techniques described above using the usual image charge construction. ${ }^{35-41}$ However, if the simulation cell is bounded by two polarizable surfaces, the situation becomes much more difficult since the image construction results in an infinite set of image charges. Therefore, both metallic ${ }^{13,42}$ and dielectric confinements ${ }^{22,43-45}$ make simulations substantially more difficult. A common procedure relies on the calculation of the induced surface charge at the interfaces using minimization of the electrostatic energy or using the discontinuity of displacement field. ${ }^{46-51}$ This makes the simulations very slow, restricting the system size to small number of particles. Recently, we $\mathrm{e}^{52}$ introduced an approach that does not rely on energy minimization but is restricted to metal plates only. If the dielectric contrast is not too large, dos Santos and Levin ${ }^{21}$ showed that it is possible to sum over the infinite set of image charges. The rate of convergence, however, deteriorates with the dielectric contrast, restricting the range of applicability of this method. There are also other approaches in the literature to deal with polarizable surfaces based on $2 d+h$ layer correction methods. ${ }^{53-55}$ Every approach has its own advantages and disadvantages.

In the present paper, we will introduce a general method for calculating the electrostatic energy of Coulomb systems 
confined by planar polarizable surfaces, either metallic or dielectric. The method is based on the exact solution of the Poisson equation ${ }^{56}$ using periodic Green functions with either Dirichlet or Neumann boundary conditions. The advantage of the new method is that it is very fast and easy to implement. A standard 3D Ewald summation code can, therefore, be easily adopted to study confined Coulomb systems in slab geometry. Alternatively one can combine our new method for calculating the polarization energy with any other fast algorithm for treating unpolarized slab geometry, such as the electrostatic layer correction (ELC) method. ${ }^{54}$ As an application, we will calculate the density profiles of ions confined between the charged dielectric and metal surfaces.

\section{GREEN FUNCTION}

Consider a point particle of charge $q_{i}$ at position $\mathbf{r}_{i}=\left(x_{i}\right.$, $y_{i}, z_{i}$ ) inside a simulation box with sides of lengths $L_{x}, L_{y}$, and $L$; in $x, y$, and $z$ directions, respectively. This system is replicated along the $x$ and $y$ axes, generating an infinite periodic charged system of finite width $L$ in the $z$ direction. The dielectric constant in the region $0<z<L$ is $\epsilon_{w}$, while in the regions $z<0$ and $z>L$, it is $\epsilon_{c}$, see Fig. 1 . The electrostatic potential at position $\mathbf{r}=(x, y, z)$ satisfies the Poisson equation

$\nabla^{2} G\left(\mathbf{r}, \mathbf{r}_{i}\right)=-\frac{4 \pi q_{i}}{\epsilon_{w}} \sum_{m_{x}, m_{y}=-\infty}^{\infty} \delta\left(\boldsymbol{r}-\boldsymbol{r}_{i}+m_{x} L_{x} \hat{\boldsymbol{x}}+m_{y} L_{y} \hat{\boldsymbol{y}}\right)$

The periodic delta function can be expressed using the Fourier transform representation as

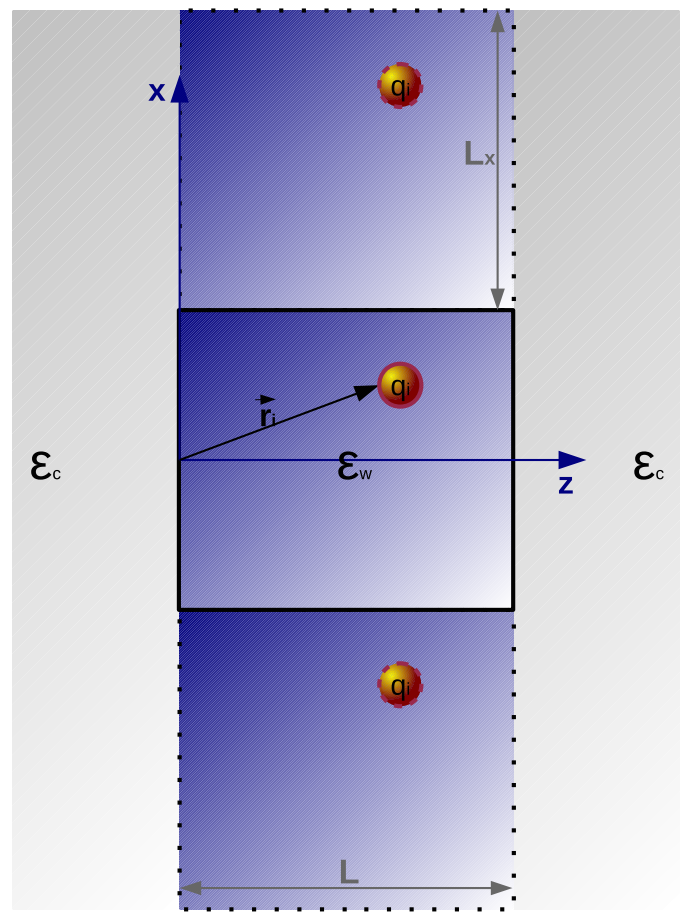

FIG. 1. Representation of the system. Only the first two images of the main simulation box in the $\hat{x}$ direction are shown.

$$
\begin{array}{r}
\sum_{m_{x}, m_{y}=-\infty}^{\infty} \delta\left(x-x_{i}+m_{x} L_{x}\right) \delta\left(y-y_{i}+m_{y} L_{y}\right) \\
=\frac{1}{L_{x} L_{y}} \sum_{\boldsymbol{m}=-\infty}^{\infty} e^{i\left[\frac{2 \pi m_{x}}{L_{x}}\left(x-x_{i}\right)+\frac{2 \pi m_{y}}{L_{y}}\left(y-y_{i}\right)\right]},
\end{array}
$$

where $\boldsymbol{m}=\left(m_{x}, m_{y}\right)$. We now write the Green function as

$$
G\left(\mathbf{r}, \mathbf{r}_{i}\right)=\frac{1}{L_{x} L_{y}} \sum_{\boldsymbol{m}=-\infty}^{\infty} g_{\boldsymbol{m}}\left(z_{i}, z\right) e^{i\left[\frac{2 \pi m_{x}}{L_{x}}\left(x-x_{i}\right)+\frac{2 \pi m_{y}}{L_{y}}\left(y-y_{i}\right)\right]},
$$

which is periodic in $\hat{\boldsymbol{x}}$ and $\hat{\boldsymbol{y}}$ directions. Inserting Eq. (3) into Eq. (1), we obtain

$$
\frac{\partial^{2} g_{\boldsymbol{m}}\left(z_{i}, z\right)}{\partial z^{2}}-k^{2} g_{\boldsymbol{m}}\left(z_{i}, z\right)=-\frac{4 \pi q_{i}}{\epsilon_{w}} \delta\left(z-z_{i}\right),
$$

where $k=2 \pi \sqrt{m_{x}^{2} / L_{x}^{2}+m_{y}^{2} / L_{y}^{2}}$. The general solution of Eq. (4) has the form $A e^{-k z}+B e^{k z}$. The electrostatic potential must vanish as $z \rightarrow \pm \infty$, restricting its form in the outer regions, $z$ $<0$ and $z>L$, to a decaying exponential. Using the symmetry properties of the Green function and the boundary conditions, we obtain

$$
\begin{aligned}
g_{\boldsymbol{m}}\left(z_{i}, z\right)= & \frac{2 \pi q_{i}}{\epsilon_{w} k\left(1-\gamma^{2} e^{-2 k L}\right)} \\
& \times\left[e^{-k\left|z-z_{i}\right|}+\gamma e^{-k\left(z+z_{i}\right)}+\gamma e^{-2 k L} e^{k\left(z+z_{i}\right)}\right. \\
& \left.+\gamma^{2} e^{-2 k L} e^{k\left|z-z_{i}\right|}\right]
\end{aligned}
$$

where $\gamma=\left(\epsilon_{w}-\epsilon_{c}\right) /\left(\epsilon_{w}+\epsilon_{c}\right)$. The periodic Green function assumes the form

$$
\begin{aligned}
G\left(\mathbf{r}, \mathbf{r}_{i}\right)= & \frac{1}{L_{x} L_{y}} \sum_{m} g_{m}\left(z_{i}, z\right) \\
& \times \cos \left[\frac{2 \pi m_{x}}{L_{x}}\left(x-x_{i}\right)+\frac{2 \pi m_{y}}{L_{y}}\left(y-y_{i}\right)\right] .
\end{aligned}
$$

In the absence of dielectric contrast, $\gamma \rightarrow 0$, Eq. (6) reduces to

$$
\begin{aligned}
G_{0}\left(\mathbf{r}, \mathbf{r}_{i}\right)= & \frac{2 \pi q_{i}}{\epsilon_{w} L_{x} L_{y}} \sum_{m=-\infty}^{\infty} \frac{e^{-k\left|z-z_{i}\right|}}{k} \\
& \times \cos \left[\frac{2 \pi m_{x}}{L_{x}}\left(x-x_{i}\right)+\frac{2 \pi m_{y}}{L_{y}}\left(y-y_{i}\right)\right],
\end{aligned}
$$

which is a representation of the electrostatic potential produced by a point charge periodically replicated in the $x$ and $y$ directions. Equation (7) diverges in the limit $k \rightarrow 0$, when $m_{x}, m_{y} \rightarrow 0$. Although this divergence can be renormalized, the remaining sum is still slowly convergent. We note, however, that the electrostatic potential described by Eq. (7) can be efficiently calculated using a modified 3D Ewald summation technique ${ }^{32,57}$ or other methods. ${ }^{9,54}$ The details of the 3D Ewald summation technique are presented in the Appendix. With the aid of Eq. (7), we can rewrite the total electrostatic potential as

$$
G\left(\mathbf{r}, \mathbf{r}_{i}\right)=\left[G\left(\mathbf{r}, \mathbf{r}_{i}\right)-G_{0}\left(\mathbf{r}, \mathbf{r}_{i}\right)\right]+G_{0}\left(\mathbf{r}, \mathbf{r}_{i}\right) .
$$

We define $\tilde{G}\left(\mathbf{r}, \mathbf{r}_{i}\right)=G\left(\mathbf{r}, \mathbf{r}_{i}\right)-G_{0}\left(\mathbf{r}, \mathbf{r}_{i}\right)$ as the polarization contribution to the total Green function given by 


$$
\begin{aligned}
\tilde{G}\left(\mathbf{r}, \mathbf{r}_{i}\right)= & \frac{2 \pi q_{i}}{\epsilon_{w} L_{x} L_{y}} \sum_{m=-\infty}^{\infty} \frac{1}{k\left(1-\gamma^{2} e^{-2 k L}\right)} \\
& \times\left[\gamma e^{-k\left(z+z_{i}\right)}+\gamma e^{-2 k L} e^{k\left(z+z_{i}\right)}\right. \\
& \left.+2 \gamma^{2} e^{-2 k L} \cosh \left(k\left(z-z_{i}\right)\right)\right] \\
& \times \cos \left[\frac{2 \pi m_{x}}{L_{x}}\left(x-x_{i}\right)+\frac{2 \pi m_{y}}{L_{y}}\left(y-y_{i}\right)\right] .
\end{aligned}
$$

The limit $k \rightarrow 0, m_{x}=m_{y}=0$, requires additional care. For -1 $<\gamma<1$, we find that the $m_{x}=m_{y}=0$ diverges as

$$
-\frac{4 \pi q_{i}}{\epsilon_{w} L_{x} L_{y}}\left[\frac{\gamma}{k(\gamma-1)}+\frac{\gamma L}{(\gamma-1)^{2}}+\mathcal{O}(k)\right] .
$$

Since this is a constant, it will not contribute to the force and can be renormalized away. For $\gamma=-1$, we find that $m_{x}=m_{y}$ $=0$ term contains an infinite constant and a finite function of $z$,

$$
\frac{2 \pi q_{i}}{\epsilon_{w} L_{x} L_{y}}\left[-\frac{1}{k}+\left(z+z_{i}-2 \frac{z_{i} z}{L}\right)+\mathcal{O}(k)\right] .
$$

Once again neglecting the infinite constant, we write

$$
G_{(-1)}\left(\mathbf{r}, \mathbf{r}_{i}\right)=\frac{2 \pi q_{i}}{\epsilon_{w} L_{x} L_{y}}\left(z+z_{i}-2 \frac{z_{i} z}{L}\right) .
$$

For $\gamma=1$, we find

$$
\frac{2 \pi q_{i}}{\epsilon_{w} L_{x} L_{y}}\left[\frac{2}{L k^{2}}-\frac{1}{k}+\frac{2 L^{2}-3 L\left(z+z_{i}\right)+3\left(z^{2}+z_{i}^{2}\right)}{3 L}+\mathcal{O}(k)\right]
$$

so that

$$
G_{(+1)}\left(\mathbf{r}, \mathbf{r}_{i}\right)=\frac{2 \pi q_{i}}{\epsilon_{w} L_{x} L_{y}}\left[-\left(z+z_{i}\right)+\frac{z^{2}+z_{i}^{2}}{L}\right] .
$$

The final expression for the total electrostatic potential can now be written as

$$
\begin{aligned}
G\left(\mathbf{r}, \mathbf{r}_{i}\right)= & G_{0}\left(\mathbf{r}, \mathbf{r}_{i}\right)+G_{(\gamma)}\left(\mathbf{r}, \mathbf{r}_{i}\right)+\frac{2 \pi q_{i}}{\epsilon_{w} L_{x} L_{y}} \\
& \times \sum_{m^{\prime}=-\infty}^{\infty} \frac{1}{k\left(1-\gamma^{2} e^{-2 k L}\right)}\left(\gamma e^{-k\left(z+z_{i}\right)}+\gamma e^{-2 k L} e^{k\left(z+z_{i}\right)}\right. \\
& \left.+2 \gamma^{2} e^{-2 k L} \cosh \left(k\left|z-z_{i}\right|\right)\right) \\
& \times \cos \left[2 \pi\left(\frac{m_{x}}{L_{x}}\left(x-x_{i}\right)+\frac{m_{y}}{L_{y}}\left(y-y_{i}\right)\right)\right]
\end{aligned}
$$

where the function $G_{(\gamma)}\left(\mathbf{r}, \mathbf{r}_{i}\right)$ is non-zero only for $\gamma=+1$ and -1 , and the prime on $\boldsymbol{m}^{\prime}$ excludes $m_{x}=m_{y}=0$ term in the summation.

The total energy for a system of $N$ periodically replicated charged particles is then given by

$$
U=\sum_{i=1}^{N} \sum_{j=1}^{N} q_{j} \frac{G\left(\mathbf{r}_{j}, \mathbf{r}_{i}\right)}{2} .
$$

We can split the total energy into the polarization and direct Coulomb contributions

$$
U=U_{E w}+U_{p},
$$

where $U_{E w}$ is the direct Coulomb contribution,

$$
U_{E w}=\sum_{i=1}^{N} \sum_{j=1}^{N} q_{j} \frac{G_{0}\left(\mathbf{r}_{j}, \mathbf{r}_{i}\right)}{2},
$$

which can be calculated using the modified 3D Ewald summation method or any other fast algorithm, see the Appendix. The energy $U_{p}$ due to surface polarizability can be rewritten as

$$
\begin{aligned}
U_{p}= & U_{\gamma}+\frac{\pi}{\epsilon_{w} L_{d}^{2}} \sum_{\boldsymbol{m}^{\prime}} \frac{\gamma}{k\left(1-\gamma^{2} e^{-2 k L}\right)} \\
& \times\left\{f_{1}(\boldsymbol{m})^{2}+f_{2}(\boldsymbol{m})^{2}+e^{-2 k L}\left(f_{3}(\boldsymbol{m})^{2}+f_{4}(\boldsymbol{m})^{2}\right)\right. \\
& \left.+2 \gamma e^{-2 k L}\left[f_{3}(\boldsymbol{m}) f_{1}(\boldsymbol{m})+f_{2}(\boldsymbol{m}) f_{4}(\boldsymbol{m})\right]\right\},
\end{aligned}
$$

where without the loss of generality, we have set $L_{x}=L_{y}=L_{d}$. The number of integers, $\left(m_{x}, m_{y}\right)$, necessary to obtain a converged energy will depend on the lateral size of the simulation box, $L_{d}$. The contribution $U_{\gamma}$ arises from the $k \rightarrow 0$ limit and is zero if $\gamma \neq( \pm 1)$. For $\gamma=-1$, we find

$$
U_{(-1)}=-\frac{2 \pi}{L_{d}^{2}}\left[\frac{M_{z}^{2}}{L}-Q_{t} M_{z}\right],
$$

where $Q_{t}=\sum_{i=1}^{N} q_{i}$ and $M_{z}=\sum_{i=1}^{N} q_{i} z_{i}$. For $\gamma=+1$, we obtain

$$
U_{(+1)}=-\frac{2 \pi Q_{t}}{L_{d}^{2}}\left[M_{z}-\frac{\Omega_{z}}{L}\right],
$$

where $\Omega_{z}=\sum_{i=1}^{N} q_{i} z_{i}^{2}$. The $f_{i}(\boldsymbol{m})$ functions are defined as

$$
\begin{aligned}
f_{1}(\boldsymbol{m}) & =\sum_{i=1}^{N} q_{i} \cos \left[\frac{2 \pi}{L_{d}}\left(m_{x} x_{i}+m_{y} y_{i}\right)\right] e^{-k z_{i}}, \\
f_{2}(\boldsymbol{m}) & =\sum_{i=1}^{N} q_{i} \sin \left[\frac{2 \pi}{L_{d}}\left(m_{x} x_{i}+m_{y} y_{i}\right)\right] e^{-k z_{i}}, \\
f_{3}(\boldsymbol{m}) & =\sum_{i=1}^{N} q_{i} \cos \left[\frac{2 \pi}{L_{d}}\left(m_{x} x_{i}+m_{y} y_{i}\right)\right] e^{k z_{i}}, \\
f_{4}(\boldsymbol{m}) & =\sum_{i=1}^{N} q_{i} \sin \left[\frac{2 \pi}{L_{d}}\left(m_{x} x_{i}+m_{y} y_{i}\right)\right] e^{k z_{i}} .
\end{aligned}
$$

Note that $k$ depends on $\boldsymbol{m}$, and the $f$ functions must be updated for each particle move. There is, however, no need to recalculate all the functions but only the contribution to each function that depends on the position of the particle that is being moved. This makes the energy update very efficient. Furthermore, the prefactors that depend on the exponential functions of $m_{x}$ and $m_{y}$ can be precalculated at the beginning of the simulation. Finally, if there is a surface charge present at the interfaces, it can be included as an external potential, see the Appendix and Ref. 57,

$$
U_{s u r}=-\frac{2 \pi\left(\sigma_{1}-\sigma_{2}\right)}{\epsilon_{w}} \sum_{i=1}^{N} q_{i} z_{i},
$$

where $\sigma_{1}$ and $\sigma_{2}$ are the surface charge densities at $z=0$ and $z=L$, respectively.

\section{SIMULATIONS AND RESULTS}

To demonstrate the utility of the new simulation method, we perform Monte Carlo simulations of an electrolyte solution 


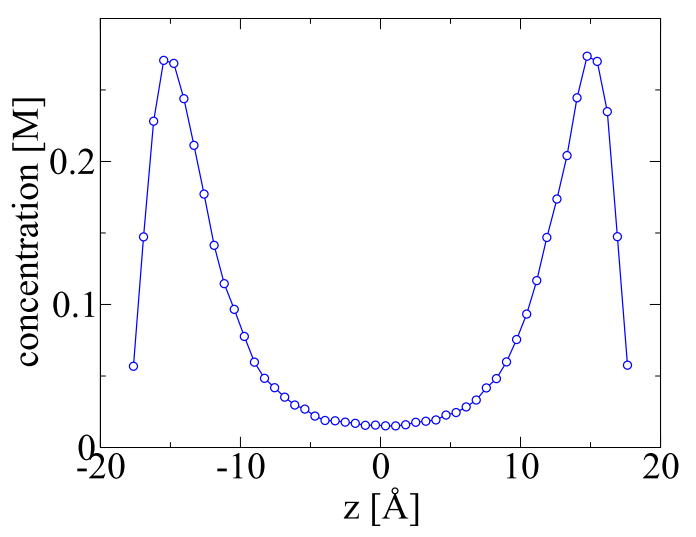

FIG. 2. Density profile of trivalent counterions confined between charged dielectric surfaces, $\gamma=0.95$. The surface charge densities are $-0.05 \mathrm{C} / \mathrm{m}^{2}$. The line is a guide to the eyes.

in the $N V T$ ensemble using the Metropolis algorithm. ${ }^{58}$ To efficiently sample the phase space, we use both short- and long-displacement moves. ${ }^{1,2}$ The effective ionic radii are set to $r_{c}=2 \AA$. The Bjerrum length, defined as $\lambda_{B}=q^{2} \beta / \epsilon_{w}$, where $\beta$ is the inverse thermal energy and $q$ is the proton charge, is set to $\lambda_{B}=7.2 \AA$, typical value for water at room temperature. The system relaxes to equilibrium in $1 \times 10^{6}$ Monte Carlo steps. The ionic density profiles are obtained using $1 \times 10^{5}$ uncorrelated samples.

In Fig. 2, we show the density profile of trivalent counterions confined between charged dielectric surfaces of $\gamma$ $=0.95$. The confining surfaces are separated by a distance $L$ $=40 \AA$. The number of counterions is $N_{c}=100$, and the surfaces are equally charged with charge density $-0.05 \mathrm{C} / \mathrm{m}^{2}$. We see a strong repulsion of ions from the interface produced by the induced surface charge. This result is in agreement with an earlier image charge algorithm. ${ }^{21}$ However, the present method is an order of magnitude more efficient.

In Fig. 3, we show the density profiles of cations and anions of a dissolved 3:1 electrolyte at concentration 0.35 $\mathrm{M}$, confined by grounded metal electrodes, $\gamma=-1$, separated by distance $L=30 \AA$. Now, instead of the repulsion of the previous case, we see the expected attraction of charges to the metal electrodes. This effect can be understood

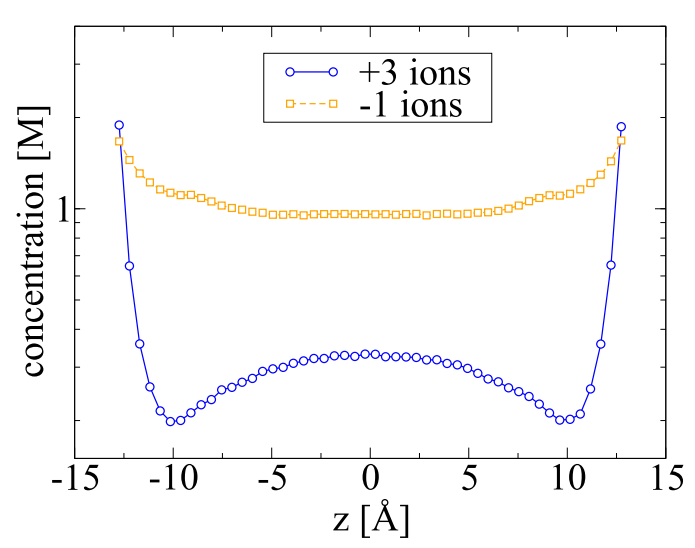

FIG. 3. Density profiles of cations and anions confined between grounded metal surfaces, $\gamma=-1$. The $3: 1$ salt concentration is $0.35 \mathrm{M}$. The lines are a guide to the eye.

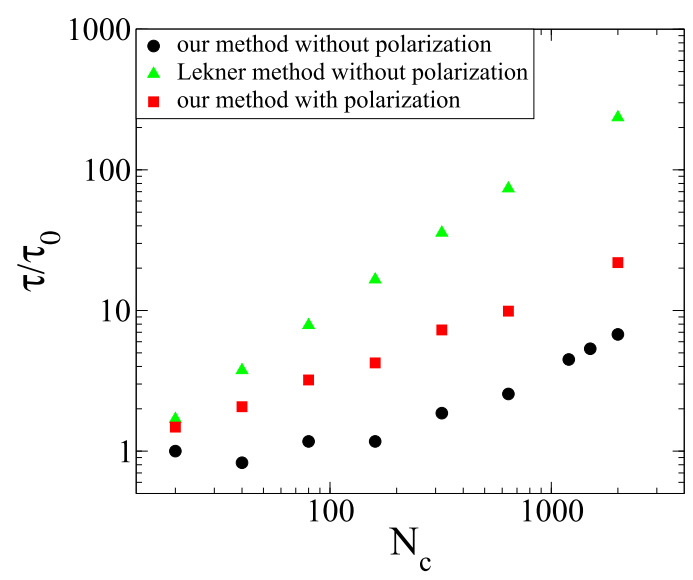

FIG. 4. CPU time to perform $10^{6}$ energy updates as a function of the number of particles in the system. The distance between the polarizable plates is $L$ $=10 \AA$, with $\gamma=0.95$. The Bjerrum length was set to $\lambda_{B}=14.5 \AA$, the superficial charge to $\sigma=-0.12 \mathrm{C} / \mathrm{m}^{2}$, and ionic radius to $2 \AA$.

considering the image charges of opposite sign induced inside the electrodes.

Finally, in Fig. 4, we compare the characteristic central processing unit (CPU) times of our simulation method with a standard implementation of the Lekner summation which does not account for polarization. ${ }^{9}$ We see that for reasonably large system sizes, the Lekner summation is at least an order of magnitude slower than our method. Furthermore, for large $N_{c}$, we see that even for systems with polarization, our method remains an order of magnitude faster than the Lekner summation without polarization. One can probably optimize our method further by combining it with a more efficient algorithm for calculating electrostatic energy of a non-polarized slab. ${ }^{54}$

\section{CONCLUSIONS}

We have presented an efficient new method for simulating Coulomb systems confined by polarizable surfaces. The method relies on the exact solution of the Poisson equation in terms of periodic Green functions. We were able to separate the electrostatic energy into polarization and direct Coulomb contributions. The latter can be efficiently calculated using a modified Ewald method developed in the previous work ${ }^{57}$ or any other fast method such as electrostatic layer correction (ELC). ${ }^{54}$ The polarization energy is separated into terms which can be locally updated for each particle move without the need for recalculating the whole electrostatic energy. The results of the new simulation method were compared with the earlier approach ${ }^{21}$ and found to lead to identical ionic density profiles, with a significant gain in simulation time. Finally, we note that the calculations presented in this paper can be easily extended to study systems with two confining walls of distinct dielectric constants.

\section{ACKNOWLEDGMENTS}

This work was partially supported by the CNPq, CAPES, Alexander von Humboldt Foundation, INCT-FCx, and by the U.S.-AFOSR under the Grant No. FA9550-16-1-0280. 


\section{APPENDIX: EWALD SUMMATION IN SLAB GEOMETRY}

For systems in slab geometry, without dielectric discontinuities, there are well-established algorithms. ${ }^{32,33,59-64}$ Recently, we developed an efficient algorithm ${ }^{57}$ where the surface charge at the slab boundaries is treated as an external potential, speeding up the traditional simulations in which the surface charges are modeled by point particles. We briefly discuss how this modified Ewald method can be used to calculate the electrostatic potential produced by a periodically replicated point charge. We start by considering an isotropic system replicated in all three dimensions and then take the slab geometry limit, in which one of the directions grows much slower than the other two. Consider $N$ particles of charge $\mathrm{q}^{j}$ confined in a cell of lengths $L_{x}, L_{y}$, and $L_{z}$. The infinite system is constructed with the definition of the replication vector $\boldsymbol{r}_{\text {rep }}=\left(n_{x} L_{x}, n_{y} L_{y}, n_{z} L_{z}\right)$, where $n$ 's span the positive and negative integers. The electrostatic potential produced by the ions and all the replicas at position $\boldsymbol{r}$ can be written as

$$
\phi(\boldsymbol{r})=\sum_{\boldsymbol{n}}^{\infty} \sum_{j=1}^{N} \int \frac{\rho^{j}(\boldsymbol{s})}{\epsilon_{w}|\boldsymbol{r}-\boldsymbol{s}|} d^{3} \boldsymbol{s}
$$

where $\rho^{j}(\boldsymbol{s})=\mathrm{q}^{j} \delta\left(\boldsymbol{s}-\boldsymbol{r}^{j}-\boldsymbol{r}_{e p}\right)$ is the charge density of $\mathrm{q}^{j}$ and its replicas. Adding and subtracting a Gaussian charge density distribution on top of each charge $\mathrm{q}^{j}$, we can split the potential into long- and short-range contributions,

$$
\begin{aligned}
\phi(\boldsymbol{r})= & \sum_{\boldsymbol{n}}^{\infty} \sum_{j=1}^{N} \int \frac{\rho_{G}^{j}(\boldsymbol{s})}{\epsilon_{w}|\boldsymbol{r}-\boldsymbol{s}|} d^{3} \boldsymbol{s} \\
& +\sum_{n}^{\infty} \sum_{j=1}^{N} \int \frac{\rho^{j}(\boldsymbol{s})-\rho_{G}^{j}(\boldsymbol{s})}{\epsilon_{w}|\boldsymbol{r}-\boldsymbol{s}|} d^{3} \boldsymbol{s},
\end{aligned}
$$

where $\rho_{G}^{j}(\boldsymbol{s})=\mathrm{q}^{j}\left(\kappa_{e}^{3} / \sqrt{\pi^{3}}\right) \exp \left(-\kappa_{e}^{2}\left|\boldsymbol{s}-\boldsymbol{r}^{j}-\boldsymbol{r}_{\text {rep }}\right|^{2}\right)$ and $\kappa_{e}$ is a damping parameter. The first term on the right-hand side of Eq. (A2) is long ranged (it has a non-integrable tail) and can be efficiently summed using the Fourier representation. The second term can be rewritten using the complementary error function. The electrostatic potential then takes the form

$$
\begin{aligned}
\phi(\boldsymbol{r})= & \sum_{\boldsymbol{k}=\mathbf{0}}^{\infty} \sum_{j=1}^{N} \frac{4 \pi \mathrm{q}^{j}}{\epsilon_{w} V|\boldsymbol{k}|^{2}} \exp \left[-\frac{|\boldsymbol{k}|^{2}}{4 \kappa_{e}^{2}}+i \boldsymbol{k} \cdot\left(\boldsymbol{r}-\boldsymbol{r}^{j}\right)\right] \\
& +\sum_{j=1}^{N} \mathrm{q}^{j} \frac{\operatorname{erfc}\left(\kappa_{e}\left|\boldsymbol{r}-\boldsymbol{r}^{j}\right|\right)}{\epsilon_{w}\left|\boldsymbol{r}-\boldsymbol{r}^{j}\right|}
\end{aligned}
$$

where $\boldsymbol{k}=\left(\frac{2 \pi}{L_{x}} n_{1}, \frac{2 \pi}{L_{y}} n_{2}, \frac{2 \pi}{L_{z}} n_{3}\right)$ and $V=L_{x} L_{y} L_{z}$, the volume of the main cell. Since the second term is short ranged, it can be treated using simple periodic boundary conditions, as long as $\kappa_{e}$ is sufficiently large.

The first term of the Fourier series diverges when $\boldsymbol{k} \rightarrow 0$. To understand better the significance of this divergence, we study this term separately by expanding it around the $k=0$. We write

$$
\begin{aligned}
\lim _{\boldsymbol{k} \rightarrow 0} & \sum_{j=1}^{N} \mathrm{q}^{j} \frac{1}{|\boldsymbol{k}|^{2}}-\sum_{j=1}^{N} \mathrm{q}^{j} \frac{1}{4 \kappa_{e}^{2}}+\lim _{\boldsymbol{k} \rightarrow 0} \sum_{j=1}^{N} \mathrm{q}^{j} \frac{i \boldsymbol{k} \cdot\left(\boldsymbol{r}-\boldsymbol{r}^{j}\right)}{|\boldsymbol{k}|^{2}} \\
& -\lim _{\boldsymbol{k} \rightarrow 0} \sum_{j=1}^{N} \mathrm{q}^{j} \frac{\left[\boldsymbol{k} \cdot\left(\boldsymbol{r}-\boldsymbol{r}^{j}\right)\right]^{2}}{2|\boldsymbol{k}|^{2}}+\mathcal{O}(|\boldsymbol{k}|) .
\end{aligned}
$$

If the system is non-neutral, it is possible to renormalize the two diverging constant terms by redefining the zero of the electrostatic potential. Consequently, we can neglect the infinite constants which do not influence the physics of the system. However, the third and fourth terms have dependence on particle positions and hence must be properly accounted for. The third sum on the right can be written as

$$
S_{3}=\sum_{j=1}^{N} \mathrm{q}^{j} \int_{-\infty}^{+\infty} \delta(\boldsymbol{k}) \frac{i \boldsymbol{k} \cdot\left(\boldsymbol{r}-\boldsymbol{r}^{j}\right)}{|\boldsymbol{k}|^{2}} d \boldsymbol{k},
$$

where we use the delta representation $\delta(\boldsymbol{k})=(2 \pi)^{-3} \int_{-\boldsymbol{H}}^{\boldsymbol{H}} e^{i \boldsymbol{k} \cdot \boldsymbol{p}}$ $d^{3} p$. The limits in delta integration, $-\mathbf{H}$ to $\mathbf{H}$, where $\mathbf{H}$ $=\left(H_{1}, H_{2}, H_{3}\right)$, must be performed in accordance with the real space sum. We define $H_{1}=\alpha_{1} L_{c}, H_{2}=\alpha_{2} L_{c}$, and $H_{3}$ $=\alpha_{3} L_{c}$, where $L_{c}$ is some characteristic macroscopic length scale. For isotropic bulk systems, $H$ 's grow at the same rate. On the other hand, for systems with slab geometry, $H_{1}$ and $H_{2}$ should grow much faster than $H_{3}$. Explicitly performing the integrals over $p$ 's we obtain

$$
\delta(\boldsymbol{k})=\frac{1}{(2 \pi)^{3}} \prod_{i=1}^{3} \int_{-\alpha_{i} \frac{L_{c}}{2}}^{\alpha_{i} \frac{L_{c}}{2}} e^{i k_{i} p_{i}} d p_{i}=\frac{1}{\pi^{3}} \prod_{i=1}^{3} \frac{\sin \left(k_{i} \alpha_{i} L_{c} / 2\right)}{k_{i}}
$$

and Eq. (A5) can now be written as $S_{3}=\sum_{j=1}^{N} q_{j} \boldsymbol{D} \cdot\left(\boldsymbol{r}-\boldsymbol{r}^{j}\right)$, where the components of the vector $\boldsymbol{D}$ are

$$
D_{n}=\frac{i}{\pi^{3}} \int_{-\infty}^{+\infty} \frac{k_{n}}{|\boldsymbol{k}|^{2}} \prod_{j=1}^{3} \frac{\sin \left(k_{j} \alpha_{j} L_{c} / 2\right)}{k_{j}} d^{3} \boldsymbol{k}
$$

which by symmetry integrates to zero, $D_{n}=0$, so that $S_{3}=0$. The last term can be written as

$$
S_{4}=-\sum_{j=1}^{N} \mathrm{q}^{j} \int_{-\infty}^{+\infty} \delta(\boldsymbol{k}) \frac{\left[\boldsymbol{k} \cdot\left(\boldsymbol{r}-\boldsymbol{r}^{j}\right)\right]^{2}}{2|\boldsymbol{k}|^{2}} d^{3} \boldsymbol{k} .
$$

Applying once again the delta function representation, we obtain

$$
S_{4}=-\sum_{j=1}^{N} \frac{\mathrm{q}^{j}}{2 \pi^{3}} \sum_{n=1}^{3} B_{n}\left(r_{n}-r_{n}^{j}\right)^{2}
$$

where the index $n$ corresponds to the $x, y$, and $z$ components of the vector $\boldsymbol{r}$ and

$$
B_{n}=\int_{-\infty}^{+\infty} d^{3} \boldsymbol{k} \frac{k_{n}^{2}}{|\boldsymbol{k}|^{2}} \prod_{j=1}^{3} \frac{\sin \left(k_{j} \alpha_{j} L_{c} / 2\right)}{k_{j}}
$$

The coefficients $B_{n}$ can be simplified to ${ }^{65}$

$$
B_{1}=\frac{\pi^{\frac{5}{2}}}{2} \int_{0}^{+\infty} \frac{\alpha_{13} e^{-\frac{\alpha_{13}^{2}}{4 t}} \operatorname{erf}\left(\frac{\alpha_{23}}{2 \sqrt{t}}\right) \operatorname{erf}\left(\frac{1}{2 \sqrt{t}}\right)}{t^{\frac{3}{2}}} d t
$$




$$
\begin{gathered}
B_{2}=\frac{\pi^{\frac{5}{2}}}{2} \int_{0}^{+\infty} \frac{\alpha_{23} e^{-\frac{\alpha_{23}^{2}}{4 t}} \operatorname{erf}\left(\frac{\alpha_{13}}{2 \sqrt{t}}\right) \operatorname{erf}\left(\frac{1}{2 \sqrt{t}}\right)}{t^{\frac{3}{2}}} d t, \\
B_{3}=\frac{\pi^{\frac{5}{2}}}{2} \int_{0}^{+\infty} \frac{e^{-\frac{1}{4 t} \operatorname{erf}\left(\frac{\alpha_{13}}{2 \sqrt{t}}\right) \operatorname{erf}\left(\frac{\alpha_{23}}{2 \sqrt{t}}\right)} d t,}{t^{\frac{3}{2}}} d t
\end{gathered}
$$

where $\alpha_{i j}=\alpha_{i} / \alpha_{j}$ are the aspect ratios of the macroscopic system. The coefficients $B_{n}$ can now be easily calculated using numerical integration. For a spherically symmetric summation of replicas, the aspect ratios are $\alpha_{13}=L_{x} / L_{z}$ and $\alpha_{23}=L_{y} / L_{z}$. On the other hand, for a plane-wise summation of slab geometry, $\alpha_{13} \rightarrow \infty$ and $\alpha_{23} \rightarrow \infty$. In this case, the integrals can be performed explicitly, ${ }^{65}$ yielding $B_{1}=B_{2}=0$ and $B_{3}=\pi^{3}$. Thus, for slab geometry, we have the renormalized electrostatic potential

$$
\begin{aligned}
\Delta \phi(\boldsymbol{r})= & \sum_{\boldsymbol{k} \neq 0}^{\infty} \sum_{j=1}^{N} \frac{4 \pi \mathrm{q}^{j}}{\epsilon_{w} V|\boldsymbol{k}|^{2}} \exp \left[-\frac{|\boldsymbol{k}|^{2}}{4 \kappa_{e}^{2}}+i \boldsymbol{k} \cdot\left(\boldsymbol{r}-\boldsymbol{r}^{j}\right)\right] \\
& -\sum_{j=1}^{N} \frac{2 \pi \mathrm{q}^{j}}{\epsilon_{w} V}\left(r_{3}-r_{3}^{j}\right)^{2}+\sum_{j=1}^{N} \mathrm{q}^{j} \frac{\operatorname{erfc}\left(\kappa_{e}\left|\boldsymbol{r}-\boldsymbol{r}^{j}\right|\right)}{\epsilon_{w}\left|\boldsymbol{r}-\boldsymbol{r}^{j}\right|},
\end{aligned}
$$

and the energy, $U_{E w}=\frac{1}{2} \sum_{i=1}^{N} \mathrm{q}^{i} \Delta \phi\left(\boldsymbol{r}^{i}\right)$, is

$$
\begin{aligned}
U_{E w}= & \sum_{\boldsymbol{k} \neq 0}^{\infty} \frac{2 \pi}{\epsilon_{w} V|\boldsymbol{k}|^{2}} \exp \left[-\frac{|\boldsymbol{k}|^{2}}{4 \kappa_{e}^{2}}\right]\left[A(\boldsymbol{k})^{2}+B(\boldsymbol{k})^{2}\right] \\
& +\frac{2 \pi}{\epsilon_{w} V}\left[M_{z}^{2}-Q_{t} \Omega_{z}\right]+\frac{1}{2} \sum_{i \neq j}^{N} \mathrm{q}^{i} \mathrm{q}^{j} \\
& \times \frac{\operatorname{erfc}\left(\kappa_{e}\left|\boldsymbol{r}^{i}-\boldsymbol{r}^{j}\right|\right)}{\epsilon_{w}\left|\boldsymbol{r}^{i}-\boldsymbol{r}^{j}\right|}-\frac{\kappa_{e}}{\epsilon_{w} \sqrt{\pi}} \sum_{i}^{N} q_{i}^{2},
\end{aligned}
$$

where

$$
\begin{aligned}
A(\boldsymbol{k}) & =\sum_{i=1}^{N} \mathrm{q}^{i} \cos \left(\boldsymbol{k} \cdot \boldsymbol{r}^{i}\right), \\
B(\boldsymbol{k}) & =-\sum_{i=1}^{N} \mathrm{q}^{i} \sin \left(\boldsymbol{k} \cdot \boldsymbol{r}^{i}\right), \\
M_{z} & =\sum_{i=1}^{N} \mathrm{q}^{i} z_{i}, \\
Q_{t} & =\sum_{i=1}^{N} \mathrm{q}^{i}, \\
\Omega_{z} & =\sum_{i=1}^{N} \mathrm{q}^{i} z_{i}^{2} .
\end{aligned}
$$

If there are surface charge densities present at the interfaces, an additional term, Eq. (26), must be included. Equation (A15) provides an efficient way of calculating the slowly converging sum in Eq. (7) allowing us to rapidly calculate the direct contribution to the total electrostatic energy, Eq. (18).

\footnotetext{
${ }^{1}$ M. P. Allen and D. J. Tildesley, Computer Simulations of Liquids (Oxford University Press, Oxford, New York, 1987).

${ }^{2}$ D. Frenkel and B. Smit, Understanding Molecular Simulation-From Algorithms to Applications (Academic Press, San Diego, California, USA, 2002), pp. 291-306.
}

${ }^{3}$ P. Ewald, "Die berechnung optischer und elektrostatischer gitterpotentiale," Ann. Phys. 369, 253-287 (1921).

${ }^{4}$ U. Essmann, L. Perera, M. L. Berkowitz, T. Darden, H. Lee, and L. G. Pedersen, "A smooth particle mesh Ewald method," J. Chem. Phys. 103, 8577-8593 (1995).

${ }^{5}$ T. Darden, D. York, and L. Pedersen, "Particle mesh Ewald: An $n \log (n)$ method for Ewald sums in large systems," J. Chem. Phys. 98, 10089-10092 (1993).

${ }^{6} \mathrm{~J}$. Kolafa and J. W. Perram, "Cutoff errors in the Ewald summation formulae for point charge systems," Mol. Simul. 9, 351-368 (1992).

${ }^{7}$ M. Deserno and C. Holm, "How to mesh up Ewald sums. I. An accurate error estimate for the particle-particle-particle-mesh algorithm," J. Chem. Phys. 109, 7678-7693 (1998).

${ }^{8}$ M. Deserno and C. Holm, "How to mesh up Ewald sums. II. An accurate error estimate for the particle-particle-particle-mesh algorithm," J. Chem. Phys. 109, 7694-7701 (1998).

${ }^{9}$ J. Lekner, "Summation of Coulomb fields in computer-simulated disordered systems," Phys. A 176, 485-498 (1991).

${ }^{10}$ A. H. Widmann and D. B. Adolf, "A comparison of Ewald summation techniques for planar surfaces," Comput. Phys. Commun. 107, 167-186 (1997).

${ }^{11}$ M. Mazars, "Lekner summations and Ewald summations for quasi-twodimensional systems," Mol. Phys. 103, 1241-1260 (2005).

${ }^{12}$ M. V. Fedorov and A. A. Kornishev, "Ionic liquids at electrified interfaces," Chem. Rev. 114, 2978-3036 (2014).

${ }^{13} \mathrm{~S}$. Reed, O. Lanning, and P. Madden, "Electrochemical interface between an ionic liquid and a model metallic electrode," J. Chem. Phys. 126, 084704 (2007).

${ }^{14}$ C. Lian, K. Liu, K. L. V. Aken, Y. Gogotsi, D. J. Wesolowski, H. L. Liu, D. E. Jiang, and J. Z. Wu, "Enhancing the capacitive performance of electric double-layer capacitors with ionic liquid mixtures," ACS Energy Lett. 1, 21-26 (2016).

${ }^{15}$ M. Dudka, S. Kondrat, A. Kornyshev, and G. Oshanin, "Phase behavior and structure of a superionic liquid in nonpolarized nanoconfinement," J. Phys.: Condens. Matter 28, 466007 (2016)

${ }^{16} \mathrm{~S}$. Coles, M. Mishin, S. Perkin, M. Fedorov, and V. Ivanistsev, "The nanostructure of a lithium glyme solvate ionic liquid at electrified interfaces," Phys. Chem. Chem. Phys. 19, 11004-11010 (2017).

${ }^{17}$ M. Girotto, T. Colla, A. P. dos Santos, and Y. Levin, "Lattice model of an ionic liquid at an electrified interface," J. Phys. Chem. B 121, 6408-6415 (2017).

${ }^{18} \mathrm{C}$. Wong and M. Muthukumar, "Polymer capture by electro-osmotic flow of oppositely charged nanopores," J. Chem. Phys. 126, 164903-164905 (2007)

${ }^{19} \mathrm{P}$. Cazade, R. Hartkamp, and B. Coasne, "Structure and dynamics of an electrolyte confined in charged nanopores," J. Phys. Chem. C 118, 5061-5072 (2014)

${ }^{20}$ S. Kondrat, N. Georgi, M. Fedorov, and A. Kornyshev, "A superionic state in nano-porous double-layer capacitors: Insights from Monte Carlo simulations," Phys. Chem. Chem. Phys. 13, 11359-11366 (2011).

${ }^{21}$ A. P. dos Santos and Y. Levin, "Electrolytes between dielectric charged surfaces: Simulations and theory," J. Chem. Phys. 142, 194104 (2015).

${ }^{22}$ T. Colla, M. Girotto, A. P. dos Santos, and Y. Levin, "Charge neutrality breakdown in confined aqueous electrolytes: Theory and simulation," J. Chem. Phys. 145, 094704 (2016).

${ }^{23}$ Z. Luo, Y. Xing, Y. Ling, A. Kleinhammes, and Y. Wu, "Electroneutrality breakdown and specific ion effects in nanoconfined aqueous electrolytes observed by NMR," Nat. Commun. 6, 6358-6365 (2015).

${ }^{24} \mathrm{P}$. Linse and V. Lobaskin, "Electrostatic attraction and phase separation of like-charged colloidal particles," Phys. Rev. Lett. 83, 4208-4211 (1999).

${ }^{25} \mathrm{M}$. Hatlo and L. Lue, "Electrostatic interactions of charged bodies from the weak-to the strong-coupling regime,” Europhys. Lett. 89, 25002 (2010).

${ }^{26}$ L. Šamaj and E. Trizac, "Counterions at highly charged interfaces: From one plate to like-charge attraction," Phys. Rev. Lett. 106, 078301 (2011).

${ }^{27}$ R. Netz and H. Orland, "Beyond Poisson-Boltzmann: Fluctuation effects and correlation functions," Europhys. J. E 1, 203-214 (2000).

${ }^{28}$ A. Martin-Molina, J. G. Ibarra-Armenta, E. Gonzalez-Tovar, R. HidalgoAlvarez, and M. Quesada-Perez, "Monte Carlo simulations of the electrical double layer forces in the presence of divalent electrolyte solutions: Effect of the ion size," Soft Matter 7, 1441-1449 (2011).

${ }^{29}$ A. Grosberg, T. Nguyen, and B. Shklovskii, "Colloquium: The physics of charge inversion in chemical and biological systems," Rev. Mod. Phys. 74, 329-345 (2002). 
${ }^{30} \mathrm{Z}$. Wang and $\mathrm{J}$. Wu, "Ion association at discretly-charged dielectric interfaces: Giant charge inversion," J. Chem. Phys. 147, 024703 (2017).

${ }^{31}$ Y. Levin, "Electrostatic correlations: From plasma to biology," Rep. Prog. Phys. 65, 1577 (2002).

${ }^{32}$ I. C. Yeh and M. L. Berkowitz, "Ewald summation for systems with slab geometry," J. Chem. Phys. 111, 3155-3162 (1999).

${ }^{33}$ M. Kawata and M. Mikami, "Rapid calculation of two-dimensional Ewald summation," Chem. Phys. Lett. 340, 157-164 (2001).

${ }^{34}$ A. Arnold, J. de Joannis, and C. Holm, "Electrostatics in periodic slab geometries. I," J. Chem. Phys. 117, 2496-2502 (2002).

${ }^{35} \mathrm{~T}$. Nagy, D. Henderson, and D. Boda, "Simulation of an electrical double layer model with a low dielectric layer between the electrode and the electrolyte,” J. Phys. Chem. B 115, 11409-11419 (2011).

${ }^{36}$ Z.-Y. Wang and Z. Ma, "Examining the contributions of image-charge forces to charge reversal: Discrete versus continuum modeling of surface charges," J. Chem. Theory Comput. 12, 2880-2888 (2016).

${ }^{37}$ M. Girotto, A. P. dos Santos, and Y. Levin, "Interaction of charged colloidal particles at the water-air interface," J. Phys. Chem. B 120, 5817-5822 (2016).

${ }^{38}$ G. Guerrero-García, Y. Jing, and M. de la Cruz, "Enhancing and reversing the electric field at the oil-water interface with size-asymmetric monovalent ions," Soft Matter 9, 6046-6052 (2013).

${ }^{39}$ A. Bakhshandeh, A. P. dos Santos, and Y. Levin, "Weak and strong coupling theories for polarizable colloids and nanoparticles," Phys. Rev. Lett. 107, 107801 (2011).

${ }^{40}$ A. P. dos Santos, A. Bakhshandeh, and Y. Levin, "Effects of the dielectric discontinuity on the counterion distribution in a colloidal suspension," J. Chem. Phys. 135, 044124 (2011).

${ }^{41}$ A. Diehl, A. P. dos Santos, and Y. Levin, "Surface tension of electrolyteair interface: A Monte Carlo study," J. Phys.: Condens. Matter 24, 284115 (2012).

${ }^{42}$ J. I. Siepmann and M. Sprik, "Influence of surface topology and electrostatic potential on water/electrode systems," J. Chem. Phys. 102, 511-524 (1995).

${ }^{43}$ A. P. dos Santos, M. Girotto, and Y. Levin, "Simulations of polyelectrolyte adsorption to a dielectric like-charged surface," J. Phys. Chem. B 120, 10387-10393 (2016).

${ }^{44}$ J. Zwanikken and M. de la Cruz, "Tunable soft structure in charged fluids confined by dielectric interfaces," Proc. Natl. Acad. Sci. U. S. A. 110, 5301-5308 (2013).

${ }^{45}$ V. Jadhao, F. Solis, and M. de la Cruz, "Simulation of charged systems in heterogeneous dielectric media via a true energy functional," Phys. Rev. Lett. 109, 223905 (2012).

${ }^{46}$ D. T. Limmer, C. Merlet, M. Sallane, D. Chandler, P. A. Madden, R. van Roij, and B. Rotenberg, "Charge fluctuations in nanoscale capacitors," Phys. Rev. Lett. 111, 106102-106106 (2013).

${ }^{47}$ C. Merlet, D. T. Limmer, M. Salanne, R. van Roij, P. A. Madden, D. Chandler, and B. Rotenberg, "The electric double layer has a life of its own,” J. Phys. Chem. C 118, 18291-18298 (2014).
${ }^{48}$ Y. Jing, V. Jadhao, J. Zwanikken, and M. de La Cruz, "Ionic structure in liquids confined by dielectric surfaces," J. Chem. Phys. 143, 194508 (2015).

${ }^{49}$ D. Boda, D. Gillespie, W. Nonner, D. Henderson, and B. Eisenberg, "Computing induced charges in inhomogeneous dielectric media: Application in a Monte Carlo simulation of complex ionic systems," Phys. Rev. E 69, 046702 (2004).

${ }^{50}$ Z. Gan, H. Wu, K. Barros, Z. Xu, and E. Luijten, "Comparison of efficient techniques for the simulation of dielectric objects in electrolytes," J. Comput. Phys. 291, 317-333 (2015).

${ }^{51}$ S. Tyagi, M. Süzen, M. Sega, M. Barbosa, S. Kantarovitch, and C. Holm, "An iterative, fast, linear-scaling method for computing induced charges on arbitrary dielectric boundaries," J. Chem. Phys. 132, 154112 (2010).

${ }^{52}$ M. Girotto, A. P. dos Santos, and Y. Levin, "Simulations of ionic liquids confined by metal electrodes using periodic green functions," J. Chem. Phys. 147, 074109 (2017).

${ }^{53}$ A. Arnold, K. Breitsprecher, F. Fahrenberger, S. Kesselheim, O. Lenz, and C. Holm, "Efficient algorithms for electrostatic interactions including dielectric contrasts," Entropy 15, 4569-4588 (2013).

${ }^{54} \mathrm{~S}$. Tyagi, A. Arnold, and C. Holm, "Electrostatic layer correction with image charges: A linear scaling method to treat slab $2 \mathrm{D}+\mathrm{h}$ systems with dielectric interfaces," J. Chem. Phys. 129, 204102 (2008).

${ }^{55}$ S. Tyagi, A. Arnold, and C. Holm, "ICMMM2D: An accurate method to include planar dielectric interfaces via image charge summation," J. Chem. Phys. 127, 154723 (2007).

${ }^{56}$ J. D. Jackson, Classical Electrodynamics (Wiley, 1999), pp. 140-141.

${ }^{57}$ A. P. dos Santos, M. Girotto, and Y. Levin, "Simulations of Coulomb systems with slab geometry using an efficient 3D Ewald summation method," J. Chem. Phys. 144, 144103 (2016).

${ }^{58}$ N. Metropolis, A. W. Rosenbluth, M. N. Rosenbluth, A. H. Teller, and E. Teller, "Equation of state calculations by fast computing machines," J. Chem. Phys. 21, 1087-1092 (1953).

${ }^{59}$ J. Hautman and M. L. Klein, "An Ewald summation method for planar surfaces and interfaces," Mol. Phys. 75, 379-395 (1992).

${ }^{60}$ E. Spohr, "Effect of electrostatic boundary conditions and system size on the interfacial properties of water and aqueous solutions," J. Chem. Phys. 107, 6342-6348 (1997).

${ }^{61}$ J. Lekner, "Summation of dipolar fields in simulated liquid-vapour interfaces," Phys. A 157, 826-838 (1989).

${ }^{62}$ R. Sperb, "Extension and simple proof of Lekner's summation formula for Coulomb forces," Mol. Phys. 13, 189-193 (1994).

${ }^{63} \mathrm{~A}$. Arnold and C. Holm, "A novel method for calculating electrostatic interactions in 2D periodic slab geometries," Chem. Phys. Lett. 354, 324-330 (2002).

${ }^{64}$ P. Mináry, M. Tuckerman, K. Pihakari, and G. Martyna, "A new reciprocal space based treatment of long range interactions on surfaces," J. Chem. Phys. 116, 5351-5362 (2002).

${ }^{65}$ E. R. Smith, "Electrostatic energy in ionic crystals," Proc. R. Soc. A 375, 475-505 (1981). 